\title{
Measurement of Vitamin D and Antioxidants Levels in Discoid Lupus Erythematosus Patients
}

\author{
Dina F. Elessawi ${ }^{(1) \#}$, Nashwa K. Radwan ${ }^{(2)}$ and I. H. Bassyouni ${ }^{(3)}$ \\ ${ }^{1)}$ Internal Medicine Unit; ${ }^{2)}$ Dermatology Unit, Health Radiation Research Department, \\ National Center for Radiation Research and Technology (NCRRT), Atomic Energy \\ Authority (AEA) and ${ }^{3}$ Rheumatology \& Rehabilitation Department, Faculty of \\ Medicine, Cairo University, Cairo, Egypt.
}

D

SCOID Lupus Erythematosus (DLE) is a chronic dermatological disease that can lead to scarring, hair loss, and hyperpigmentation changes in skin if it is not treated early and promptly. Diagnosis of discoid lupus is generally made based on clinical features. Sometimes, tests are required, including blood and urine samples. Similar to other many autoimmune conditions, DLE is generally a lifelong condition. However, there are treatments available that are usually effective and can help keep symptoms under control. IL-17 isoforms (IL-17A and IL-17F) are implicated in DLE.

Active Vitamin D3 $\left(1,25(\mathrm{OH})_{2 \mathrm{D} 3}\right)$ is a fat-soluble vitamin that its principle action is to increase the absorption of calcium and phosphate ions from the intestine and directly affect the calcification process. Beside its crucial role in calcium metabolism and bone remodeling, active vitamin D3 is considered an immunomodulator. Increased reactive oxygen species (ROS), lipid peroxides, and low antioxidant status occur in autoimmune disorder.

This study aims to assess the level of vitamin D3 [25(OH) D3] in Discoid Lupus Erythematosus (DLE) patients compared to normal controls, its correlation with disease activity parameters, laboratory parameters and antioxidants levels.

Twenty patients with (DLE) and 20 healthy controls matched for age and gender were included into this prospective study. Serum vitamin D3 $[25(\mathrm{OH}) \mathrm{D} 3]$ is measured as it is the precursor of active vitaminD3 $\left[1,25(\mathrm{OH})_{2 \mathrm{D} 3}\right]$ which has a very short half-life. Oxidative stress was assessed by measuring serum malondialdehyde (MDA) and enzymatic antioxidant status by estimating superoxide dismutase (SOD) and glutathione peroxidase (GPX).

The results of the present work are highly suggestive of the importance of vitamin D3 supplementation in (DLE) and the importance of topical antioxidants to control flare in DLE patients.

The serum levels of vitamin D3, SOD, and GPX were significantly lower in DLE patients when compared to their levels in the control group $(\mathrm{p}<0.000)$.On the other hand, MDA was significantly higher in the DLE patients than that in the healthy control subjects $(p<0.001)$. A highly statistical negative correlation between vitamin D3 with MDA level $(\mathrm{P}<0.004$ and $\mathrm{r}=$ $-0.615)$ was observed. On the other hand, SOD and GPX positively correlated with vitamin D3 level ( $\mathrm{P}<0.001$ and $\mathrm{P}<0.000$ respectively and $\mathrm{r}=0.680$ and 0.734 respectively).

It could be suggested that low vitamin D3 and disturbed antioxidant levels are prevalent in the Egyptian DLE patients. There is a clear association between vitamin D3 and antioxidants with different signs of disease activity. A routine vitamin D3 and antioxidant supplementations are recommended to DLE patients for better prognosis.

Keywords: Discoid Lupus Erythematosus/Vitamin D3 / Antioxidants.

"Correspondsing author email: dinaelessawi@hotmail.com

DOI : 10.21608/ejrsa.2017.2023.1027

(C) 2017 The National Information and Documentation Center (NIDOC) 
Cutaneous Lupus Erythematosus (CLE) can be divided into three main subtypes: acute, subacute, and chronic, all of them demonstrate photosensitivity. Acute Cutaneous Lupus Erythematosus (ACLE) most commonly presents as symmetric erythema overlying the malar cheeks and nasal bridge with sparing of the nasolabial folds (butterfly rash) (Kindle et al., 2016).

However, it can also present as a diffuse morbilliform eruption with erythema and edema of the hands, with prominent sparing of the joints. Subacute cutaneous lupus erythematosus (SCLE) characteristically presents as annular or psoriasiform plaques in a photodistribution. (Debu et al., 2014).

The most common specific skin manifestation of lupus is discoid LE(DLE), and its presence is one of the eleven American College of Rheumatology diagnostic criteria for SLE (Tan et al., 1982). DLE represents up to $80 \%$ of all cutaneous lupus cases (Gronhagen et al., 2011), and this skin manifestation is notable for its tendency to cause disfigurement, alopecia, and scarring (Rothfield et al., 1963). Approximately 1 in 4 patients with SLE has DLE (Sanchez et al., 2011), and, in some DLE cases, the skin can be the only end-organ affected without extracutaneous involvement. Interestingly, a recent observational, populationbased study of over 1000 cutaneous lupus patients revealed an overall rate of progression to SLE of $18 \%$ within a three year follow-up period (Gronhagen et al., 2011). The ease of sampling the affected skin potentially makes DLE a convenient and accessible model to study endorgan pathology in SLE. DLE classically presents with erythematous-to-violaceous, scaly plaques with prominent follicular plugging that often results in scarring and atrophy. DLE may occur in the presence or the absence of systemic disease, (Tao et al., 2012).

Lupus erythematosus is a polygenic autoimmune disease linked to various HLA subtypes, immune signaling, and environmental factors, which ultimately leads to autoantibody production and T-cell dysfunction. However, the exact etiology of DLE is not well understood. DLE likely occurs in genetically predisposed individuals, but the exact genetic connection has not been determined yet (Eastham and Vleugels, 2014).

It has been suggested that a heat-shock protein is induced in the keratinocyte following ultraviolet (UV) light exposure or stress, and this protein may act as a target for gamma (delta) T-cell-mediated epidermal cell cytotoxicity. Additionally, toll-like receptors may be involved in the pathogenesis (Merola et al., 2013).

Although the prognosis of patients with DLE is favorable regarding mortality, morbidity can be considerable. Patients may experience pain or burning of their lesions, and many experience disfigurement from the scars or atrophy that can develop. Scarring alopecia is particularly disturbing for patients. Prompt treatment of early lesions may help prevent or lessen the severity of scarring and atrophy (Chasset et al., 2015).

Exacerbation is common with increased sun exposure, particularly in the spring and summer. Ocurrence of a serious systemic disease is rare, but when it occurs, patients may develop lifealtering sequelae. Malignant degeneration within DLE lesions is uncommon (Wahie et al., 2011).

Therapy with sunscreens, topical corticosteroids, and antimalarial agents is often effective. However, immunosuppressive and/or immunomodulatory agents may be required for recalcitrant disease (Kindle et al., 2016).

Some families may carry genes that increase the risk of developing DLE. However, it is not entirely clear how the affected genes do this, or to what degree they influence the disease. It is thought that a combination of environmental factors and genetics most likely contribute to the development of DLE (Debu et al., 2014).

Similar to many autoimmune conditions, DLE is generally a lifelong condition. However, there are treatments available that are usually effective and can help keep symptoms under control (Tao et al., 2012).

Vitamin D3 in its physiologically active form (active vitamin D3) has immunoregulatory activities in addition to being a hormone controlling of the intestinal absorption of calcium and phosphate ions. Its role in the immune system may lead to the prevention of autoimmune diseases when adequate serum levels of vitamin D3 are maintained. The serum level of vitamin D3 may also have an effect on the outcome of treatment in DLE patients (Cutolo et al, 2009).

Vitamin D3 receptor has been detected in the immune system such as mononuclear cells, 
dendritic cells, and antigen-presenting cells and activated T-B lymphocytes. In addition, activated dendritic cells produce vitamin D3. (Arnson, et al., 2007).

Vitamin D shows an evidence relating to the health effects of serum Vitamin D levels, sunlight exposure and Vitamin D intake remains inconclusive. Avoiding sunlight exposure for patients suffering from light sensitivity, reduces the risk of melanoma and other skin cancers that may be associated with_Vitamin D deficiency. Individuals avoiding all sun exposure should consider having their serum Vitamin D measured. If levels are reduced or deficient they may wish to consider taking supplementary vitamin D3, 10-25 micrograms per day, and increasing their intake of foods high in Vitamin D such as oily fish, eggs, meat, fortified margarines and cereals. Vitamin D3 supplements are widely available in health food shops (Chasset et al., 2015).

Antioxidants level reflects the imbalance between the systemic manifestation of reactive oxygen species and a biological system's ability to readily detoxify the reactive intermediates or to repair the resulting damage. Disturbances in the normal redox state of cells can cause toxic effects through the production of peroxides and free radicals that damage all components of the cell, including proteins, lipids, and DNA. Further, some reactive oxidative species act as cellular messengers in redox signaling. Thus, disturbed antioxidants level can cause disruptions in normal mechanisms of cellular signaling (Pratviel and Genevieve, 2012).

Disturbed antioxidants level is caused by the over prevalence of free radicals inside the human body. These free radicals are everywhere and there is no way escaping them. These problems create a lifetime of other problems such as social or financial collapse. Disturbed antioxidants level has been proven to be a direct link to autoimmune disease and is said be a root cause of this problem (Shaabani, et al., 2009).

There is much evidence that antioxidant team that covers glutathione reductase, catalase, glutathione peroxidase, superoxide dismutase, and glucose-6-phopshate destroy reactive oxygen species and other free radicals through enzymatic as well as non-enzymatic means. The change in relative levels of antioxidants and/or free radical formation could be used as indicators for effective and earlier diagnosis of autoimmune disorders
(Karunasinghe, 2006).

Aim of Work:

In the present study, the assessment of the level of vitamin D3 in DLS patients compared to normal controls, laboratory parameters and antioxidants levels were measured. Antioxidants level was assessed by measuring serum malondialdehyde (MDA) and enzymatic antioxidant status by measuring activities of superoxide dismutase (SOD) and glutathione peroxidase (GPX).

\section{Subjects and Methods}

Twenty DLE patients and 20 healthy controls matched for age and gender were included into this prospective study. All patients and controls were subjected to the following: full history taking, complete physical examination and routine investigations. They were recruited from the outpatient clinic of Rheumatology and Rehabilitation, Kasr El Aini, Cairo University and the clinic of Rheumatology and Rehabilitation and clinic of Dermatology at the NCRRT, Atomic Energy Authority, during the period from April 2017 till July 2017.

All the patients were diagnosed clinically by two independent dermatologists evaluated the DLE .

Exclusive criteria: they are not taking any medications that can affect the results of study.

Vitamin D3 was determined by Radio immune Assay (RIA) Kit for its quantitative level in serum (Kirk and Linda, 2007).

Cellular Antioxidants Superoxide dismutase was measured by colorimetric method (Nishikimi, et al., 1972) glutathione peroxidase UV Method ( Paglia and Valentine , 1967) and malondialdehydecolorimetric Method (Ohkawa, et al.,1979 ).

\section{Results}

Twenty DLE patients and 20 healthy controls matched for age and gender were included into this prospective study. The diagnosis of discoid lupus is generally determined based on clinical features. Skin manifestation is notable for its tendency to cause disfigurement, alopecia, and scarring.

DLE classically presents with erythematousto-violaceous, scaly plaques with prominent follicular plugging that often results in scarring and atrophy. DLE may occur in the presence or 
the absence of systemic disease.

\section{Patients' Characteristics}

The disease duration of (DLE) patients ranges between 1 year to 3 years. The laboratory parameters were presented in Table 1.

Serum vitamin D3 and antioxidant levels in DLE patients and Healthy Controls

Table 2 and Figures (1), (2a), (2b) and (2c) show a Comparison between DLE patients and healthy controls regarding the mean concentration of the studied parameters. The serum levels of vitamin D3, SOD, and GPX were significantly lower in DLE patients when compared to their levels in the control group $(p<0.000)$. On the other hand, levels of MDA were significantly higher in the DLE patients than their levels in the healthy control subjects (p-value $<0.000)$.

TABLE 1. Routine Laboratory parameters of the DLE patients $\left(\mathrm{No}_{\mathbf{0}}=\mathbf{2 0}\right)$.

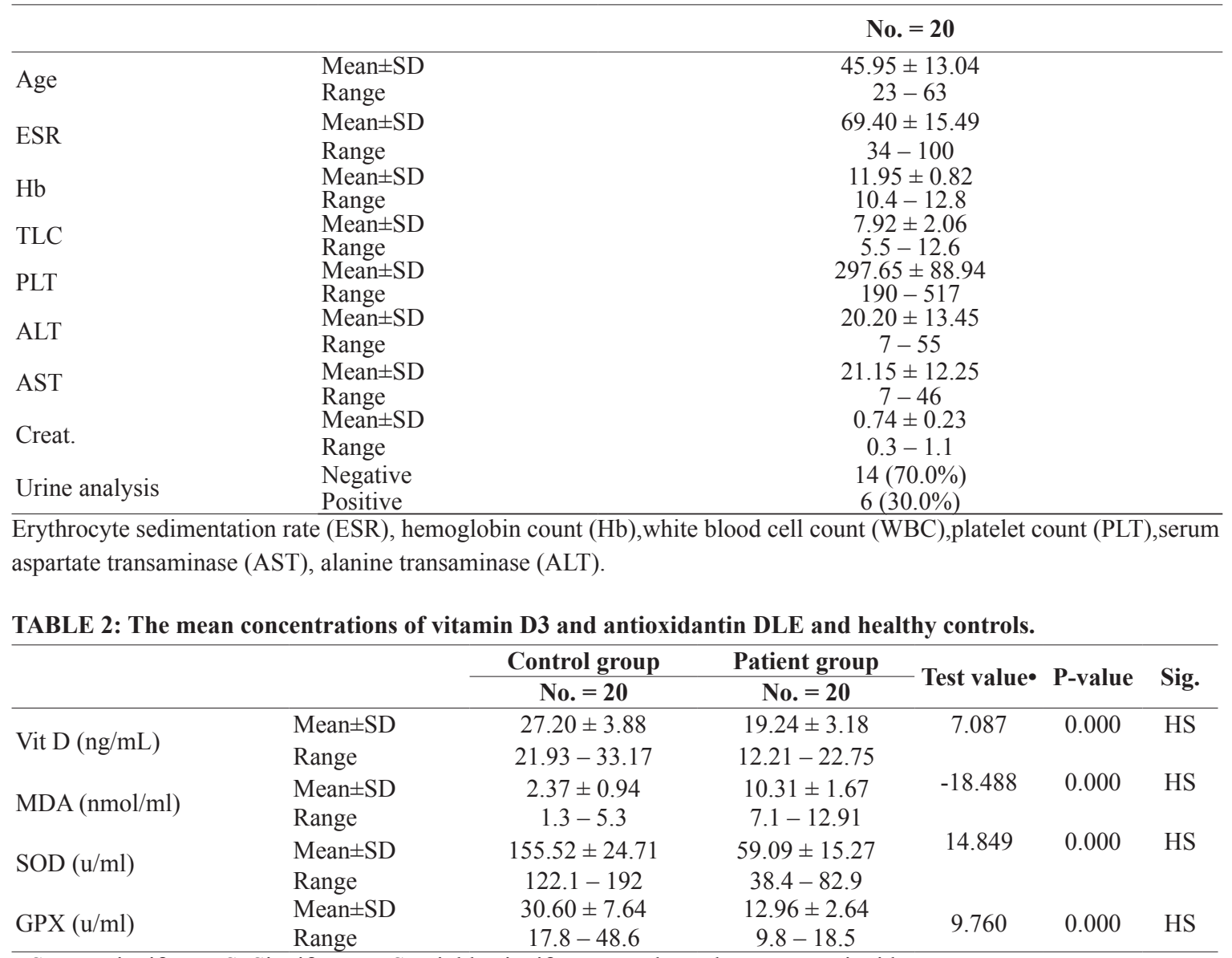

NS: Non significant; S: Significant; HS: Highly significant $\bullet$ : Independent t-test antioxidant.

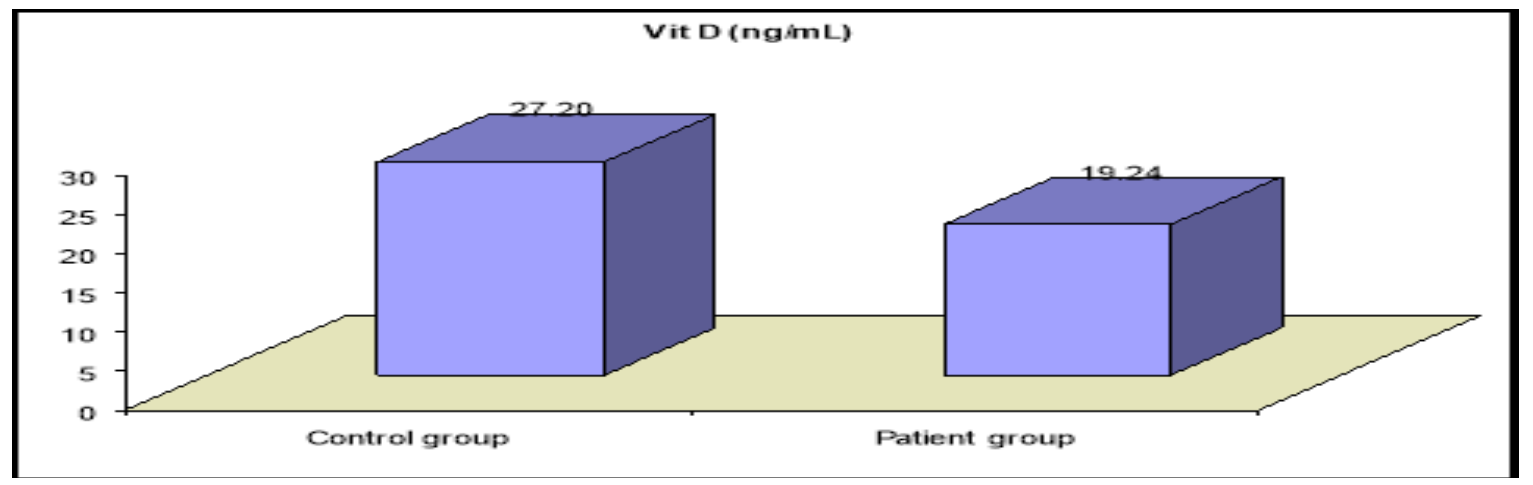

Fig.1. Serum vitamin D3 levels patients and healthy controls. 


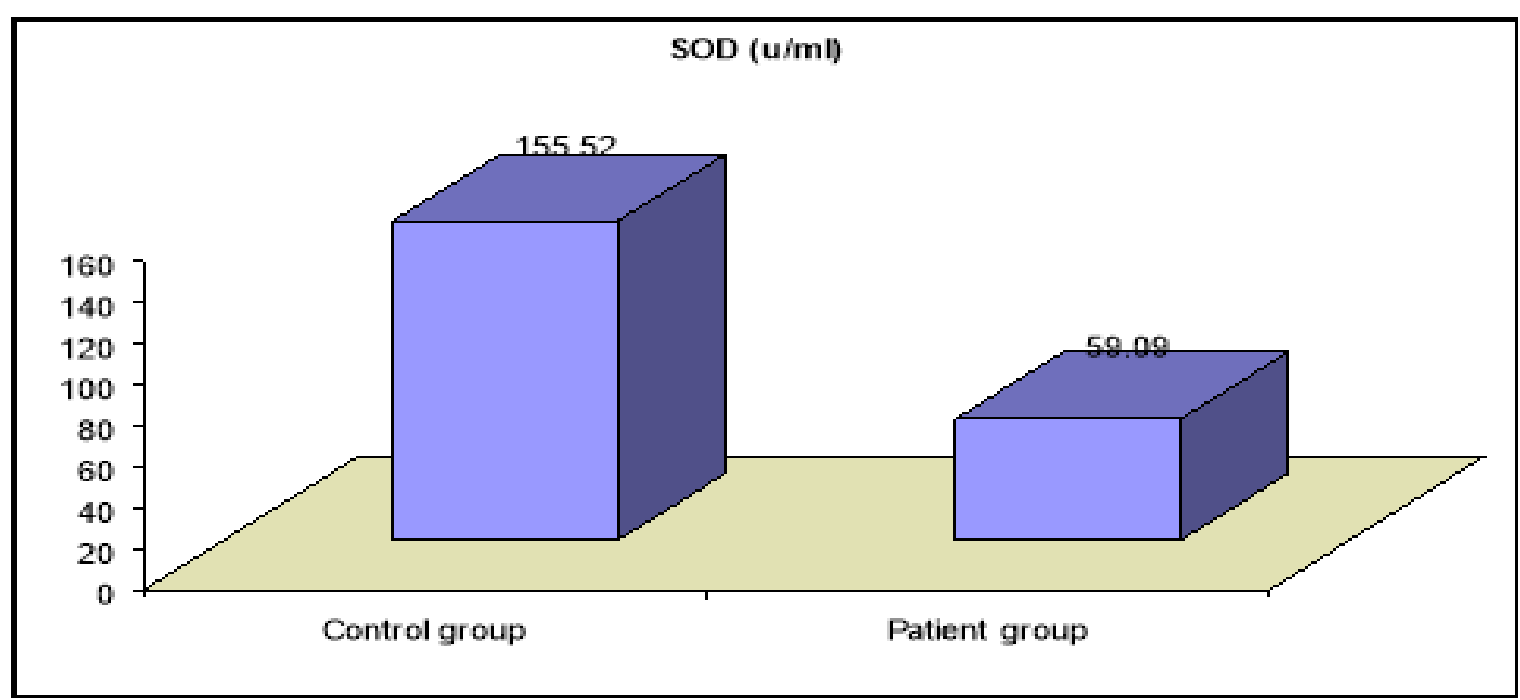

Fig. 2a . Serum antioxidant(SOD) levels in DLE patients and healthy controls

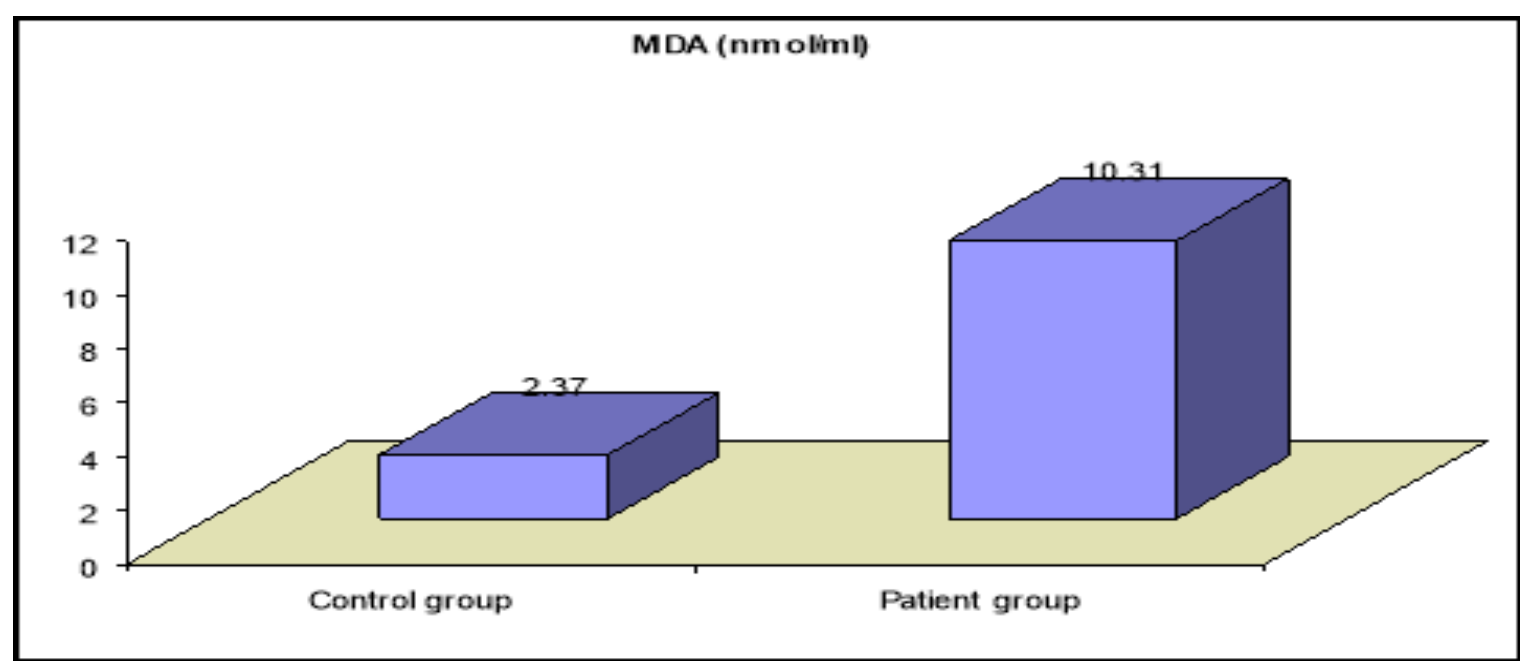

Fig.(2b). Serum antioxidantlevels (MDA) in DLE patients and healthy controls.

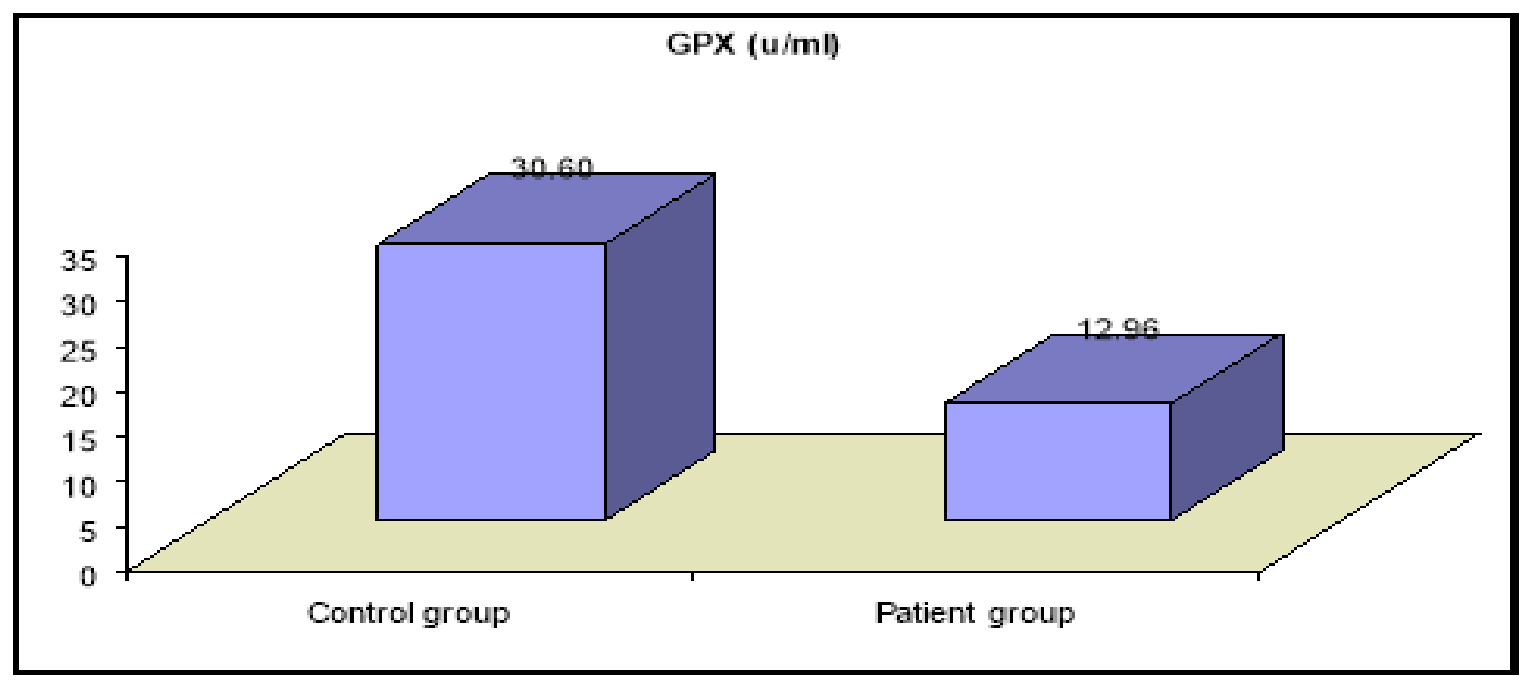

Fig. 2c. Serum antioxidantlevels(GPX) in DLE patients and healthy controls 
There is a significant negative correlation between vitamin D3 level and ESR level. On the other hand, total calcium concentrations positively correlated with the vitamin D3 levels ( $\mathrm{p}=0.001)$.

Erythrocyte sedimentation rate (ESR), hemoglobin count $(\mathrm{Hb})$, white blood cell count (WBC), platelet count (PLT), Calcium (total and free) (ca),serum aspartate transaminase (AST), alanine transaminase (ALT).
The correlation of vitamin D3 with antioxidants levels were presented in Table 4 and Fig. 4 and 5. A highly statistical negative correlation between vitamin D3 with MDA levels $(\mathrm{P}<0.004)$ was observed. On the other hand, SOD and GPX positively correlated with vitamin D3.levels $(\mathrm{P}<0.001$ and $\mathrm{P}=0.000$ respectively).

TABLE 3. Correlation of concentrations of vitamin D3 with Laboratory parameters in DLE Patients.

\begin{tabular}{lcc}
\hline & \multicolumn{2}{c}{ Vit D (ng/mL) } \\
\hline Age & r & P-value \\
Hb & -0.054 & 0.822 \\
TLC & 0.153 & 0.519 \\
PLT & 0.238 & 0.313 \\
ALT & -0.150 & 0.528 \\
AST & -0.283 & 0.226 \\
Creat. & -0.197 & 0.404 \\
ESR & -0.097 & 0.685 \\
\hline Spearmans & $-0.676^{* *}$ & 0.001 \\
\hline
\end{tabular}

Spearman's rank correlation was used, ${ }^{*} p<0.05$

Erythrocyte sedimentation rate (ESR), hemoglobin count (Hb), white blood cell count (WBC), platelet count (PLT),Calcium (total and free) (ca),serum aspartate transaminase (AST), alanine transaminase (ALT).

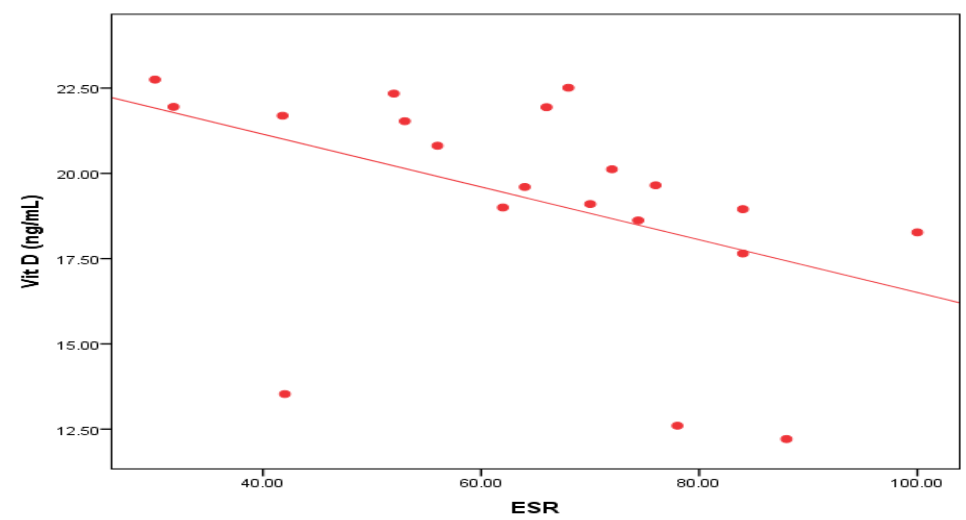

Fig. 3. Correlation between, ESR and vitamin D3

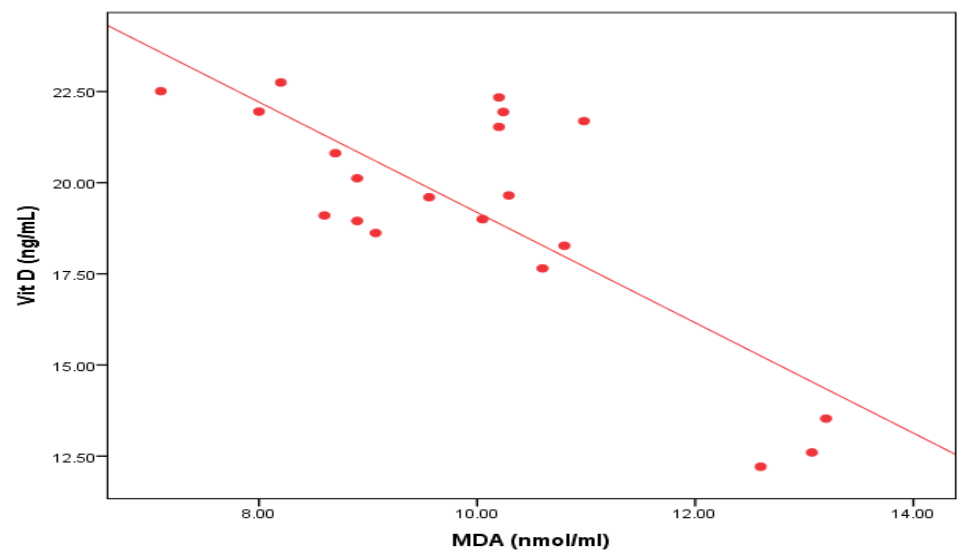

Fig. 4. Correlation between vitamin D3 with MDA 
TABLE 4. Correlation of concentrations of vitamin D3 with antioxidant levels in DLE Patients.

\begin{tabular}{lcc}
\hline & \multicolumn{2}{c}{ Vit D $(\mathbf{n g} / \mathbf{m L})$} \\
\cline { 2 - 3 } & $\mathbf{r}$ & P-value \\
\hline MDA $(\mathrm{nmol} / \mathrm{ml})$ & $-0.615^{* *}$ & 0.004 \\
$\operatorname{SOD}(\mathrm{u} / \mathrm{ml})$ & $0.680^{* *}$ & 0.001 \\
GPX $(\mathrm{u} / \mathrm{ml})$ & $0.734^{* *}$ & 0.000 \\
\hline Spearman's rank correlation was used *p $<0.05$ &
\end{tabular}

Spearman's rank correlation was used, ${ }^{*} \mathrm{p}<0.05$
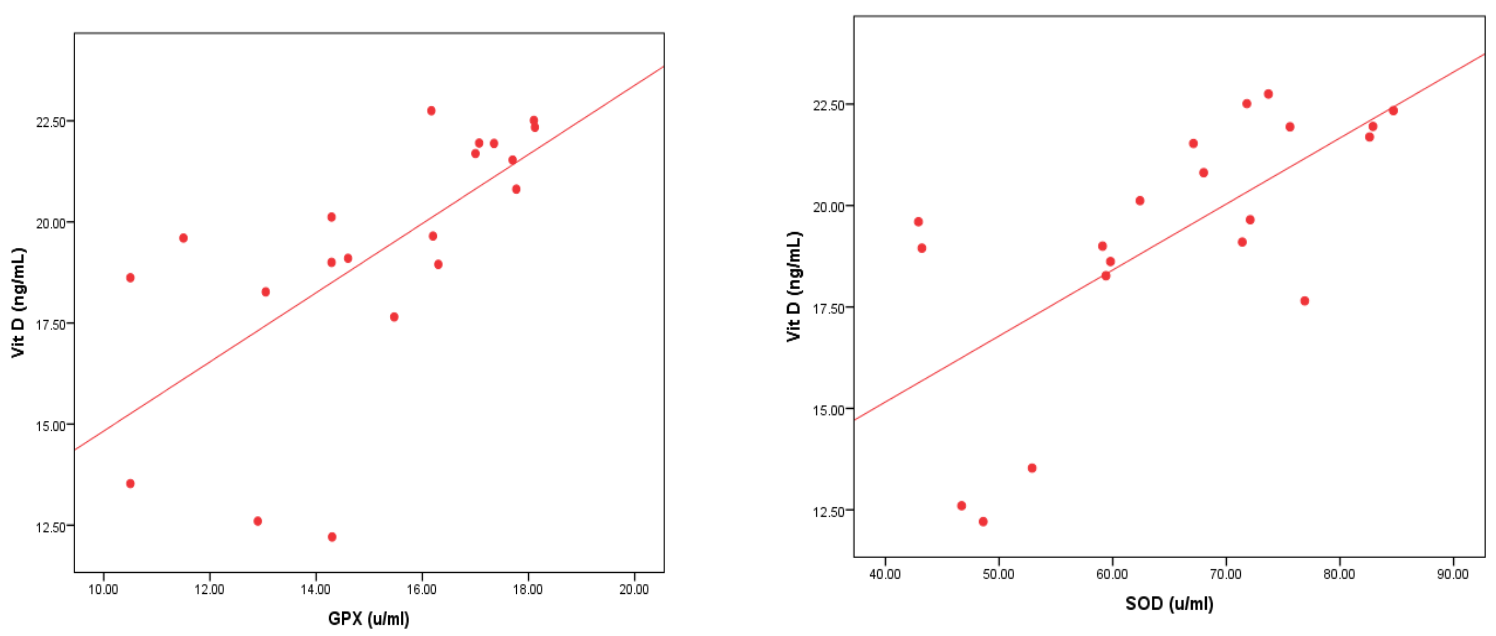

Fig. 5.Correlation between vitamin D3 with GPX and SOD

\section{Discussion}

DLE is a cutaneous manifestation of lupus that causes scarring and disfigurement. Treatment usually requires systemic immunosuppressive agents with ill-defined mechanisms of action. The potential for harmful side effectsrequires a frequent laboratory surveillance. In many cases, available systemic agents are unable to adequately control the disease. There is, therefore, a critical need for the development of a targeted therapeutic agent with a favorable side effect profile (Nestle et al., 2009).

The rational identification of potential therapeutic targets requires a well-developed understanding of the pathogenesis of disease. Psoriasis is an example of an autoimmune skin disease in which our growing understanding of the cytokines and $\mathrm{T}$ cells critical to the disease have led to the identification of new treatment targets. Biologics that inhibit IL-17- and IL-22mediated signaling, recently identified to play critical roles in this disease (Nestle et al., 2009), are at various stages of development (Leonardi et al., 2012; Papp et al., 2012), with some now entering the final phases of clinical trials.
A molecular characterization of DLE, however, has not been carried out to the same extent as that for psoriasis. The current knowledge of DLE pertains to targeted assessments of specific signaling pathways and has been most well described for type I interferons (Braunstein et al., 2012; Wenzel et al., 2005a; Wenzel et al., 2005b; Wenzel et al., 2009). Cytokines associated with specific $\mathrm{T}$ cell subsets have been explored in two separate prior studies (Stein et al., 1997; Toro et al., 2000), although their results conflicted with each other and were published prior to the discovery of the Th17 and Th22 T cell subsets.

Recent descriptions of the presence of Th17 cells in the blood of DLE patients (Balanescu et al., 2010) as well as a growing literature on the role of Th17 cells in SLE (Balanescu et al., 2010; Chen et al., 2010; Henriques et al., 2010; Kleczynska et al., 2011; Mok et al., 2010; Shah et al., 2010) seemed to support the hypothesis that Th17 cells would be present at appreciable numbers and may play a role in the development of discoid lesions The data of the present study illustrate that minimal Th17 involvement in DLE lesions were unexpected. Several reports have described the presence of IL-17 related activity or signals 
in DLE lesions by immunohistochemistry (Oh et al., 2011; Tanasescuet al., 2010) or in the serum of DLE patients (Balanescu et al., 2010). IL-17related markers have also been reported as up regulated compared to healthy controls in the serum of SLE patients in some studies (Shah et al., 2010; Zhao et al., 2010), but not all (Brajac et al., 2010; Higgs et al., 2012). In a few cases, enhanced numbers of Th17 cells significantly increased only in those patients with higher SLEDAI scores, a research instrument that integrates clinical data and laboratory results to assess SLE activity and identify SLE flares (Dolff et al., 2010).

Vitamin D3 plays an important role, along with the essential minerals calcium and phosphorus, in the maintenance of healthy bones and teeth. In addition to its regulatory role in calcium balance and bone metabolism, vitamin D3 has immunomodulatory effects that help maintain immune homeostasis. (Arnson et al., 2007).

The present study reveals that the serum levels of vitamin D3 was significantly lower $(\mathrm{P}<0.001)$ in DLE patients when compared to their level in control group (Table2 and Fig1). These results confirm the observation of common vitamin D3 deficiencies in Egyptian patients with established DLE. This observation could be interpreted in the view of many roles played by vitamin D3 in the immune system.

The hypothesis that vitamin D3 relates to autoimmune disorders emerged from people living near the equator where a decreased risk of developing common autoimmune diseases .The latitude related prevalence of autoimmune diseases coincides with the area where vitamin D deficiency is proved (Pelajo, et al., 2010).

Epidemiological evidence indicates a significant association between vitamin D3 deficiency and an increased incidence of autoimmune diseases and clarification of the physiological role of endogenous vitamin D receptors (VDR) agonists in the regulation of autoimmune responses will support the pharmacological VDR agonists for use in the clinic.(Higgins et al., 2013).

The anti-proliferative, pro-differentiative, immunomodulatory and anti-inflammatory properties of synthetic VDR agonists could be exploited to treat a variety of autoimmune rheumatic diseases, from Rheumatoid arthritis to Systemic lupus erythematosis and possibly also multiple sclerosis, type 1 diabetes (Shoenfeld et al., 2009).

Furthermore, the active vitamin D3 analogs protect against the development of autoimmune encephalomyelitis in murine mice especially when combined with other immunosuppressants (Pelajo et al., 2010).

The obtained results of measuring vitamin D3 levels indicate a high significant negative correlation between vitamin D3 levels with parameters of DLE disease activity, especially ESR ( $\mathrm{r}=0.382, \mathrm{p}=0.006$ ) (Table 3). Vitamin D3 inversely related to DLE disease activity, the low vitamin D3 intake increases the risk of developing autoimmune disorders in most published series (Song et al., 2012).

Many studies were conducted to investigate the influence of vitamin D3 level on the activity of Systemic Lupus Erythematosis in Egyptian patients. Vitamin D 3 deficiencies is highly prevalent among patients with disease activity compared to the patients in remission. Also, vitamin D level correlated inversely with disease activity, which suggests that inadequate vitamin D level, among other factors, probably contributed to the development of active disease in patients with SLE (Fahmi, et al., 2014),( Kamel, et al., 2017), (Shereen Algergawy ,2017), ( Kamal, et al., 2015), (Nouran, et al., 2016).

In addition, the greater intake of vitamin D3 is inversely related to the risk of developing RA. The RA disease activity assessed by DAS 28 could be influenced by vitamin D intake. However, it is difficult to know whether this is due to a true immunomodulatory effect of vitamin D3 or more subjective effect of low vitamin D3 on pain perception (Higgins et al., 2013).

On the other hand, no significant correlation was observed between vitamin D3 with laboratory parameters (Table 3).

An attempt to understand the role of reactive oxygen species in pathogenesis of DLE and protection were carried out by measuring the antioxidant enzyme activities (superoxide dismutase; SOD and Glutathione peroxidase GPX) and also the assessment of serum malondialdhyde(MDA) in DLE patients and the control people. The obtained results showed significant decreases $(\mathrm{P}<0.001)$ in antioxidant enzymes activities (SOD and GPX) and a significant increase $(\mathrm{P}<0.001)$ in the level of 
serum MDA in DLE patients compared to their levels in healthy control subjects (Table 2) and (Figure (2a), (2b), (2c).

The decrease in antioxidant parameters (SOD, GPX) and increases in proxidant; MDA might be sustained the oxidative stress associated with DLE establishment. Oxidative stress occurred when the natural antioxidant system failed to cope with free radicals over production in the biological system.

Calcium deficiency and low bone mineral density is common in patients with DLE. A study examined the use of calcium and vitamin D3 supplements in patients with autoimmune disorders revealed that $1,000 \mathrm{mg}$ of calcium and $400 \mathrm{IU}$ of vitamin D3 significantly increased the bone mineral density in these patients. (Arifa, et al., 2012).

Specialized tests known as bone mineral density (BMD) tests measure bone density in various sites of the body. These tests can detect osteoporosis before a fracture occurs and predict one's chances of fracturing in the future. Rheumatoid arthritis (RA) and systemic lupus erythematosus (SLE) patients, particularly those receiving corticosteroid (glucocorticoid) therapy for two months or more, should talk to their doctors about whether they might be candidates for a bone density tests (Carol Eustice, 2012).

Generation of reactive oxygen species is an important factor in the development and maintenance of autoimmune disorders in humans. The study of Walwadkar et al. investigated the interplay among oxidants, antioxidants and pathogenesis of rheumatoid arthritis. They found that there is a close association between bone loss and oxidative threat in patients presenting with immune diseases specially RA (Walwadkar et al., 2006).

In the present study, total calcium concentration is positively correlated with the vitamin $\mathrm{D} 3$ levels $(\mathrm{p}=0.005)$ and $(\mathrm{r}=0.387)$.

From the results obtained, it could be concluded that low vitamin D3 and disturbance in antioxidants system participate largely in the pathogenesis of DLE in Egyptian patients. The analgesic effect of vitamin D3 supplementation could be attributed to its immunosuppressive capacity. Routine vitamin D and adjuvant antioxidant supplementation are recommended for better DLE disease prognosis and also in all immune disorders for its autoimmune modulation property.

\section{Conclusion}

Serum vitamin D levels have been found to be correlated inversely with the DLE disease activity. Greater intake of vitamin D was associated with a lower symptoms. Moreover, a significant clinical improvement was strongly correlated with the immunomodulating potential in vitamin D-treated DLE patients. The recommended dosage of vitamin D supplementation will need further study to assign the most appropriate dose and schedule of therapy. The intervention with antioxidant-enriched margarine in DLE patients results in consistent and significant relief of clinical symptoms. Furthermore, increases in blood antioxidant status and indications for effects on oxidative stress markers were observed. These data are promising and indicate the need for a double-blind, placebo-controlled randomized trial to establish effect and demonstrate causality.

\section{Refrences}

Arifa Alam Jelly, Shelina Begum, Sultana Ferdousi(2012) Serum Zinc, Copper and Calcium Levels in Female Rheumatoid Arthritic Patients. Journal of Bangladesh Society of Physiologist, $\mathrm{Vol}$ 7, No 1.

Arnson, Y.; Amital, H. and Shoenfeld Y (2007) Vitamin D and autoimmunity: new etiological and therapeutic considerations. Ann Rheum Dis; 66,1137-42.

Balanescu P, Balanescu E, Tanasescu C, Nicolau A, Tanasescu, R, Grancea, C. (2010) Thelper 17 cell population in lupus erythematosus. Rom J Intern Med. 48, 255-9.

Brajac I, Kastelan M, Perisa D, Simonic E, StojnicSosa L, Massari LP. (2010) Treatment of alopecia areata: modern principles and perspectives. Lijec Vjesn. 132, 365-9.

Braunstein, I, Klein, R, Okawa, J. and Werth, VP. (2012) The interferon-regulated gene signature is elevated in subacute cutaneous lupus erythematosus and discoid lupus erythematosus and correlates with the cutaneous lupus area and severity index score. $\mathrm{Br} J$ Dermatol. 166, 971-5.

Carol Eustice(2012)What Rheumatoid Arthritis Patients Need to Know About Osteoporosis. Rheumatoid Arthritis and Osteoporosis-Prevention and Treatment Part 2 of 2 -Updated June 19.

Chang LM, Maheshwari P, Werth S, Schaffer L, Head SR, Kovarik C. (2011)Identification and molecular 
analysis of glycosaminoglycans in cutaneous lupus erythematosus and dermatomyositis. J. Histochem Cytochem. 59, 336-45.

Chasset, F, Francès, C, Barete, S, Amoura, Z and Arnaud, L. (2015) Influence of smoking on the efficacy of antimalarials in cutaneous lupus: a metaanalysis of the literature. J Am Acad Dermatol. Apr. 72 (4), 634-9.

Chen XQ, Yu YC, Deng HH, Sun JZ, Dai Z, Wu YW, (2010) Plasma IL-17A is increased in new-onset SLE patients and associated with disease activity. $J$ Clin Immunol. 30, 221-5.

Cutolo, M.; Otsa, K.; Paolino, S., Yprus, M., Veldi, T. and Seriolo, B.(2009)Vitamin D involvement in rheumatoid arthritis and Systemic lupus erythaematosus. Ann Rheum Dis; 68, 446-7.

Debu A, Girard C, Bessis and D. Discoid (2014) lupus erythematosus successfully treated by photodynamic therapy. Br J. Dermatol. Sep 12.

Dolff S, Quandt D, Wilde B, Feldkamp T, Hua F, Cai $X$, (2010) Increased expression of costimulatory markers CD134 and CD80 on interleukin-17 producing $\mathrm{T}$ cells in patients with systemic lupus erythematosus. Arthritis research \& therapy. 12, R150.

Eastham, A.B. and Vleugels, R.A. (2014) Cutaneous lupus erythematosus. JAMA Dermatol. Mar. 150(3), 344

Fahmi E. Emam, Taghreed M Abd El-Wahab, Maha S. Mohammed, Amal S. Elsalhy, Sabah I Abdel Rahem (2014) Assessment of serum vitamin D level in patients with systemic lupus erythematosus Egyptian Rheumatology and Rehabilitation Year : 2014 | Volume : 41 | Issue : 2 | Page : 71-78

Gronhagen, C.M., Fored, C.M., Granath, F. and Nyberg F. (2011) Cutaneous lupus erythematosus and the association with systemic lupus erythematosus: a population-based cohort of 1088 patients in Sweden. Br J Dermatol. 164,1335-41.

Henriques, A., Ines, L., Couto, M., Pedreiro, S, Santos, C., Magalhaes, M. (2010) Frequency and functional activity of Th17, Tc17 and other T-cell subsets in Systemic Lupus Erythematosus. Cell Immunol. 264,97-103.

Higgs, B.W., Zhu, W., Richman, L., Fiorentino, DF, Greenberg, SA, Jallal, B. (2012) PIdentification of activated cytokine pathways in the blood of systemic lupus erythematosus, myositis, rheumatoid arthritis, and scleroderma patients. Int J. Rheum Dis., 15, 25-35.

Higgins, MJ.; Mackie, SL.; Thalayasingam, N.; Bingham, SJ.; Hamilton, J. and Kelly, CA. (2013) The Effect of vitamin D levels on the assessment of disease activity in rheumatoid arthritis. Clin Rheumatol. Jan 23.

Kamel H., Gado ,Tarek H.Gado ,Rasha M. AbdelSamie, Noha, M., Khalil, Safa, L., Emam, Hanan H., Fouad (2017) Clinical significance of vitamin D deficiency and receptor gene polymorphism in systemic lupus erythematosus patient The Egyptian Rheumatologist Volume 39, Issue 3, July, Pages 159-164

Kamal El Garf, Huda Marzouk, Yomna Farag, Laila Rasheed, Ayman El Garf (2015)Vitamin D status in Egyptian patients with juvenile-onset systemic lupus erythematosus Rheumatology InternationalSeptember 35, 9, pp 1535-1540.

Karunasinghe, N. (2006) Hemolysate thioredoxin reductase and glutathione peroxidase activities correlate with serum selenium in a group of NewZealand men at high prostate cancer risk. $J$ Nutr 136,2232-2235.

Kindle SA, Wetter DA, Davis MD, Pittelkow MR, Sciallis GF. Lenalidomide treatment of cutaneous lupus erythematosus: the Mayo Clinic experience. Int J Dermatol. 2016 Feb 12. 3 (4), 883-99.

Kirk Sujishi, Linda Post (2007) 25OH Vitamin D Total Assay - DiaSorin Liaison Ted Kurtz, Md (Parnassus \& China Basin).

Kleczynska, W., Jakiela, B., Plutecka, H., Milewski, M, Sanak, M. and Musial, J. (2011) Imbalance between Th17 and regulatory T-cells in systemic lupus erythematosus. Folia Histochem Cytobiol. 49, 646-53.

Leonardi C, Matheson R, Zachariae C, Cameron G, Li L. and Edson-Heredia E. (2012)Anti-interleukin-17 monoclonal antibody ixekizumab in chronic plaque psoriasis. N Engl J Med. 366,1190-9.

Merola JF, Prystowsky SD, Iversen C, Gomez-Puerta JA, Norton T. and Tsao P. (2013) Association of discoid lupus erythematosus with other clinical manifestations among patients with systemic lupus erythematosus. J. Am Acad Dermatol. Jul. 69 (1),19-24. 
Mok MY, Wu HJ, Lo Y and Lau CS. (2010) The relation of interleukin 17 (IL-17) and IL-23 to Th1/Th2 cytokines and disease activity in systemic lupus erythematosus. J. Rheumatol. 37, 2046-52.

Nestle FO, Kaplan DH, Barker J. Psoriasis. and N Engl J Med. 361, 496-509 (2009).

Nishikimi, M.; Roa, NA. and Yogi, K (1972) The occurrence of superoxide anion in the reaction of reduced phenazine methosulfate and molecular oxygen. Biochem. Bioph. REs. Commun., 46 (2),849-854

Nouran M.Abaza, Reem M. El-Mallah, Asmaa Shaaban, Sameh A. Mobasher, Khaled F. Al-hassanein, Amr A. Abdel Zaher, and Rania H. El-Kabarity (2016) Vitamin D Deficiency in Egyptian Systemic Lupus Erythematosus Patients: How Prevalent and Does It Impact Disease Activity? Integr Med Insights. 2016; 11, 27-33. Published online 2016 Sep 26. doi: $10.4137 /$ IMI.S40035

Oh SH, Roh HJ, Kwon JE, Lee SH, Kim JY, Choi H.J. (2011) Expression of interleukin-17 is correlated with interferon-alpha expression in cutaneous lesions of lupus erythematosus. Clin Exp Dermatol. 36, 512-20.

Ohkawa, H., Ohishi, W. and Yagi, K. (1979) Assay for lipid peroxides in animal tissues by thiobarbituric acid reaction Anal . Biochem , Jun; 95(2), 351-8.

Paglia, DE. and Valentine, WN. (1967) Studies on the quantitative and qualitative characterization of erythrocyte glutathione peroxidase. J Lab Clin Med, 70(1), 158-69.

Papp, K.A., Leonardi,C., Menter, A., Ortonne, J.P, Krueger, JG. and Kricorian, G. (2012)Brodalumab, an anti-interleukin-17-receptor antibody for psoriasis. N Engl J Med. 366,1181-9.

Pelajo, CF., Lopez-Benitez, JM. and Miller, LC. (2010) Vitamin D and autoimmune rheumatologic Disorders. Autoimmunity Reviews. 31(3), 315-324.

Pratviel and Genevieve (2012) "Oxidative DNA Damage Mediated by Transition Metal Ions and Their Complexes". InAstrid Sigel, Helmut Sigel and Roland K.O.Sigel. Interplay between Metal Ions and Nucleic Acids. Metal Ions in Life Sciences. Springer. pp. 201-216.

Rothfield, N, March CH, Miescher P, McEwen C. (1963) Chronic Discoid Lupus Erythematosus. $N$ Engl J Med. 269,1155-61.

Sanchez, E, Nadig, A, Richardson, BC, Freedman
BI, Kaufman KM, Kelly JA, (2011) Phenotypic associations of genetic susceptibility loci in systemic lupus erythematosus. Ann Rheum Dis. 70,1752-7.

Shah K, Lee WW, Lee SH, Kim SH, Kang SW Craft J, (2010) Dysregulated balance of Th17 and Th1 cells in systemic lupus erythematosus. Arthritis research \& therapy. 12,R53.

Shaabani, Y.; Foroughi, M.; Rastmanesh, R.; Jamshidi, A.; Tajik, N. and Assadi, O. (2009) Assessment of antioxidantnutrient intake and malondiadehyde plasma level in rheumatoid arthritis Atherosclerosis J; 5, 1-5.

Shereen Algergawy (2017) Vitamin D Therapy Among Egyptian Systemic Lupus Erythematosus Patients .Rheumatology, Volume 56, Issue suppl_2, 1 April kex062.296, -https://doi.org/10.1093/ rheumatology/ kex062.296

Shoenfeld N, Amital H, Shoenfeld Y (2009) The effect of melanism and vitamin D synthesis on the incidence of autoimmune disease. Nat Clin; Rheumatol Pract 5, 99-105.

Song, GG, Bae, SC.and Lee, YH (2012) Association between vitamin $\mathrm{D}$ intake and the risk of rheumatoid arthritis: a meta-analysis. ClinRheumatol 31:17339.

Stein, LF, Saed GM, Fivenson, DP. (1997) T-cell cytokine network in cutaneous lupus erythematosus. J Am Acad Dermatol. 36, 191-6.

Tan EM, Cohen AS, Fries JF, Masi AT, McShane DJ, Rothfield NF, et al. (1982) The 1982 revised criteria for the classification of systemic lupus erythematosus. Arthritis and rheumatism. ;25:1271-7.

Tanasescu C, Balanescu E, Balanescu P, Olteanu R, Badea C, Grancea C, et al. (2010) IL-17 in cutaneous lupus erythematosus. Eur J Intern Med. 21, 202-7.

Tao J, Zhang X, Guo N, et al. (2012) Squamous cell carcinoma complicating discoid lupus erythematosus in Chinese patients: review of the literature, 1964-2010. J Am Acad Dermatol. Apr. 66(4), 695-6.

Toro JR, Finlay D, Dou X, Zheng SC, LeBoit PE, Connolly MK. (2000) Detection of type 1 cytokines in discoid lupus erythematosus. Arch Dermatol. 136, 1497-501.

Wahie S, Daly AK, Cordell HJ, et al. (2011) Clinical and pharmacogenetic influences on 
response to hydroxychloroquine in discoid lupus erythematosus: a retrospective cohort study. $J$ Invest Dermatol. Oct. 131(10), 1981-6.

Walwadkar, S. D.; Suryakar, A. N. ; Katkam, R. V.; Kumbar, K. M. \&Ankush R. D.(2006) Oxidative stress and calcium-phosphorus levels in Rheumatoid arthritisIndian Journal of Clinical BiochemistrySeptember, Volume 21, pp 134-137.

Wenzel J, Uerlich M, Worrenkamper E, Freutel S, Bieber T, Tuting T. (2005a) Scarring skin lesions of discoid lupus erythematosus are characterized by high numbers of skin-homing cytotoxic lymphocytes associated with strong expression of the type I interferon-induced protein MxA. $\mathrm{Br} J$ Dermatol. 153, 1011-5.
Wenzel J, Worenkamper E, Freutel S, Henze S, Haller O, Bieber T, et al. (2005b) Enhanced type I interferon signalling promotes Th1-biased inflammation in cutaneous lupus erythematosus. $J$ Pathol. 205, $435-42$.

Wenzel J, Zahn S, Bieber T, Tuting T. (2009) Type I interferon-associated cytotoxic inflammation in cutaneous lupus erythematosus. Arch Dermatol Res. 301, 83-6.

Zhao XF, Pan HF, Yuan H, Zhang WH, Li XP, Wang $\mathrm{GH}$, et al. (2010) Increased serum interleukin 17 in patients with systemic lupus erythematosus. Mol. Biol. Rep. 37, 81-5.

(Received 13/11/2017; accepted 24/12/2017)

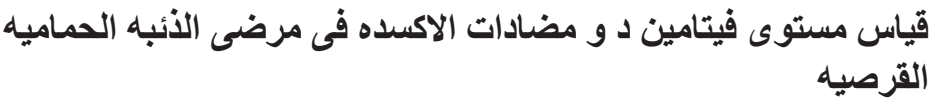

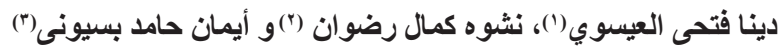

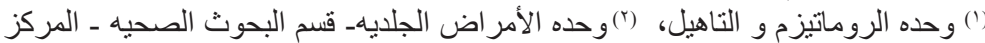

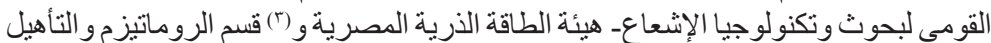

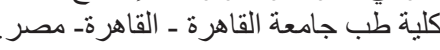

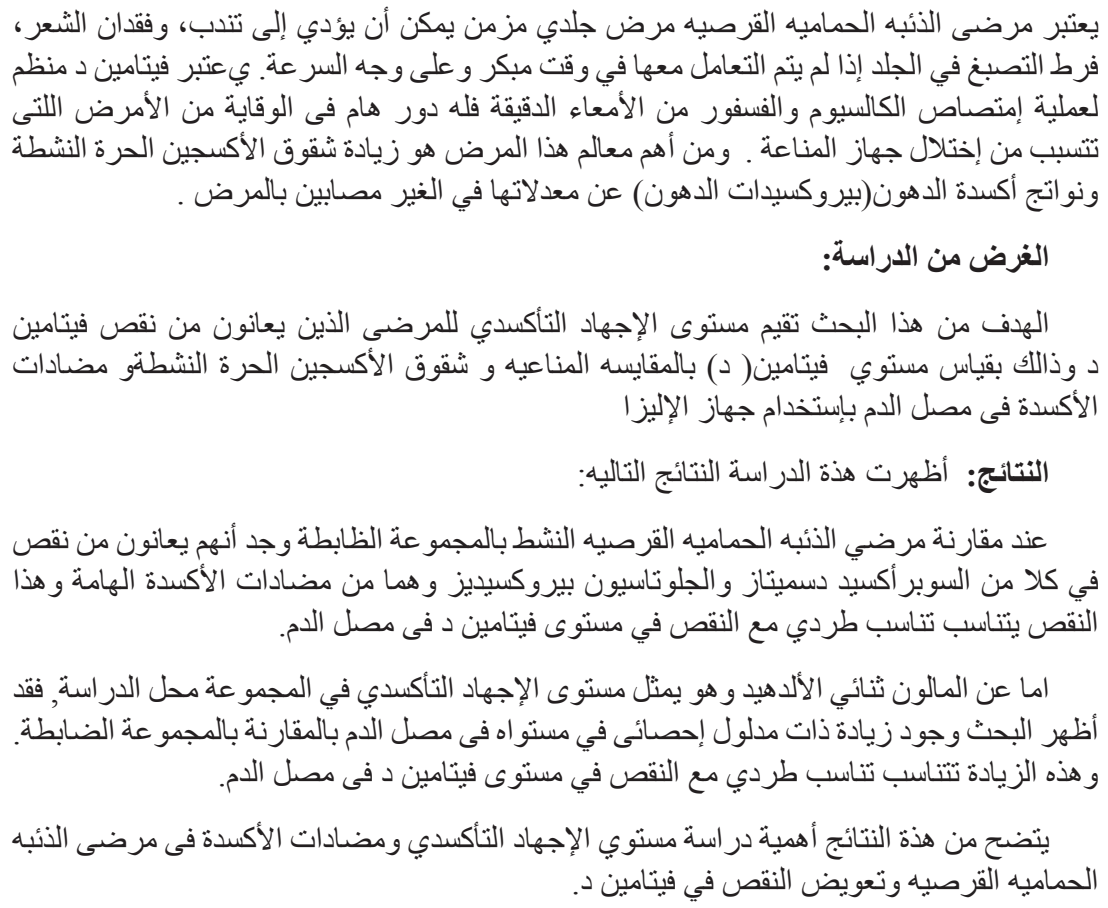

